\title{
Petit Gateau com recheio de doce de banana com e sem casca
}

\author{
Petit Gateau with banana sweet filling with and without peel \\ Rafaela Teixeira Rodrigues do Vale Costa ${ }^{1}$, Ana Marinho do Nascimento ${ }^{2}$, Alfredina dos Santos Araujo ${ }^{3}$, José Crisóstomo da \\ Silva Neto ${ }^{4}$ Cybelle de Oliveira Dantas ${ }^{5}$
}

\begin{abstract}
Resumo: A busca por alimentos alternativos, visando o reaproveitamento de resíduos que seriam descartados, bem como a agregação de valor ao produto final com redução de custos e, a melhoria na qualidade nutricional do alimento possibilitou o desenvolvimento desse estudo para elaboração e posterior análise microbiológica, sensorial e físico-química de Petit Gateau com recheio de doce de banana com e sem casca. Nas análises microbiológicas, apenas o produto com casca apresentou contaminação, $0.3 \mathrm{NMP} / \mathrm{g}$, para Coliformes a $30^{\circ} \mathrm{C}$. Para os demais parâmetros, Coliformes a $45^{\circ} \mathrm{C}$ e Salmonela sp $/ 25 \mathrm{~g}$

, ambas as formulações apresentaram-se ausentes a contaminação. Sensorialmente, frente aos atributos analisados, não foram observadas diferenças significativas entre as formulações. Quanto as características físico-químicos, os valores médios encontrados para o produto sem casca foram $62.84 \%$ para umidade, $6.98 \%$ para cinzas, 7.77 para o $\mathrm{pH}, 9.9$ para o ${ }^{\circ}$ Brix, $0.55 \%$ para acidez, $17.94 \%$ para lipídeos e $2,6 \mathrm{~N}$ para textura. Para produtos com casca, os valores encontrados nas respectivas análises foram $65.55 \%, 7.53 \%, 7.66,14.5,0.55 \%, 16.01 \%$ e $1.5 \mathrm{~N}$. Assim, Petit Gateau preparado com recheio de doce de banana com casca pode ser utilizado na indústria de alimentos como forma de minimizar desperdícios, reduzir custos e propiciar melhor qualidade nutricional do alimento.
\end{abstract}

Palavras-chaves: Alimento alternativo, reaproveitamento, bolo quente, resíduo.

\begin{abstract}
The search for alternative foods, targeting the reuse of waste that would otherwise be discarded, as well as adding value to the final product with cost reduction and the improvement in the nutritional quality of the food made possible the development of this study for elaboration and further analysis microbiological, sensory and physico-chemistry of Petit Gateau with sweet stuffing of banana with and without bark. The microbiological analysis, only the product with bark showed contamination, $0.3 \mathrm{MPN} / \mathrm{g}$, for Coliforms to $30^{\circ} \mathrm{C}$. For other parameters, the $45^{\circ} \mathrm{C}$ Coliforms and Salmonella sp $/ 25 \mathrm{~g}$, both formulations were absent the contamination. Sensorially, front of attributes analyzed, no significant differences were observed between the formulations. As the physico-chemical characteristics, the mean values found for the product without bark were $62.84 \%$ for moisture, $6.98 \%$ for ashes, 7.77 to the $\mathrm{pH}$, to $9.9^{\circ} \mathrm{Brix}, 0.55 \%$ to $17.94 \%$, acidity for lipids and $2.6 \mathrm{~N}$ for texture. For products with bark, the values found in the respective analysis was $65.55 \%, 7.53 \%, 7.66,14.5,0.55 \%, 16.01 \%$ and $1.5 \mathrm{~N}$. Thus, Petit Gateau prepared with sweet stuffing of banana with bark can be used in the food industry as a way to minimize waste, reduce costs and provide better nutritional quality of the food.
\end{abstract}

Key words: Alternative food, reuse, hot cake, residue

\footnotetext{
*Autor para correspondência

Recebido para publicação em 04/01/2015; aprovado em 20/04/2015

${ }^{1}$ Graduanda em Engenharia de Alimentos, UFCG/ CCTA/UATA, Campus Pombal-PB, e-mail: rafatrv@yahoo.com.br.

${ }^{2}$ Graduanda em Engenharia de Alimentos, UFCG/CCTA/UATA, Campus Pombal-PB, e-mail: anamarinho06@hotmail.com

${ }^{3}$ Engenharia de Processos, Professora da UFCG/CCTA/UATA, Campus Pombal-PB, e-mail: alfredina@ @cta.ufcg.edu.br

${ }^{4}$ Graduando em Engenharia de Alimentos, UFCG/CCTA/UATA, Campus Pombal-PB, e-mail: jose.engalimentos_4@hotmail.com Química Industrial, D.Sc.

${ }^{5}$ Farmacêutica, D.Sc. Ciência e Tecnologia de Alimentos, Professora da UFPB/CCHSA/DGTA, Campus Bananeiras, E-mail: cybelle.pereira@ hotmail.com
} 


\section{INTRODUÇÃO}

O nome Petit Gateau vem do francês e se refere a um pequeno bolo que geralmente é servido quente na forma de sobremesa e caracteriza-se por apresentar casca crocante, recheio cremoso e em consistência de calda. Na maioria dos casos, o sabor mais consumido da sobremesa é o de chocolate acompanhado de sorvete de creme.

Além do Petit Gateau clássico de chocolate, o bolinho pode ser encontrado em sabores como: limão, doce de leite, goiabada, laranja, chocolate branco, café, banana e outros.

No caso específico da banana (Musa spp.), vale ressaltar que independentemente de seu grupo genômico, é, sem dúvida, uma das frutas mais consumidas no mundo além de, também ser utilizada como matéria-prima de outras formulações alimentares. Trata-se de um alimento altamente energético (cerca de $100 \mathrm{kcal}$ por $100 \mathrm{~g}$ de polpa), cujos carboidratos (cerca de 22\%) são facilmente assimiláveis. Embora pobre em proteínas e lipídeos, seus teores superam os da maçã, pera, cereja ou pêssego. Contém tanta vitamina $\mathrm{C}$ quanto à maçã, além de razoáveis quantidades de vitamina $\mathrm{A}$, $\mathrm{B} 1, \mathrm{~B} 2$, pequenas quantidades de vitaminas $\mathrm{D}$ e E, e maior percentagem de potássio, fósforo, cálcio e ferro do que a maçã ou a laranja (EMBRAPA, 1997).

A alta produção e consumo de banana, resultam na produção de grande quantidade de resíduos que podem causar problemas ambientais e que poderiam ser reaproveitados na indústria de alimentos como fonte de nutrientes para alimentos alternativos.

A utilização de alimentos alternativos para o combate à fome na população de baixa renda é assunto que tem recebido atenção no Brasil nos últimos anos, especialmente em razão do drama crescente da população carente (FARFAN, 1998).

De maneira geral, o homem necessita de qualquer modo, de uma alimentação sadia, rica em nutrientes, isto pode ser alcançado com partes de alimentos que normalmente são desprezadas. Sendo assim, é importante a utilização de cascas, talos e folhas, pois o aproveitamento integral dos alimentos, além de diminuir os gastos com alimentação e melhorar a qualidade nutricional do cardápio, reduz o desperdício de alimentos e torna possível a criação de novas receitas, como, por exemplo, sucos, doces, geleias, farinhas, etc (GONDIM et al., 2005).

Segundo Gondim (2005), resíduos como cascas de alimentos apresentam teores de nutrientes muitas das vezes maiores do que os das suas respectivas partes comestíveis, além de serem ricas fontes de fibras. O valor nutricional de frutas e legumes não é nenhuma novidade. O que as pessoas não sabiam é que as cascas de alguns desses alimentos possuem nutrientes como vitamina $\mathrm{C}$, carboidratos, cálcio e fibras, muitas vezes em quantidades superiores às da própria polpa (UNESP, 2014).

Tendo em vista a crescente importância pela busca por alimentos alternativos, visando o reaproveitamento de resíduos que seriam descartados no meio ambiente, a agregação de valor ao produto final com redução de custos e, a melhora na qualidade nutricional do alimento, o objetivo desse estudo foi à elaboração e posterior análise microbiológica, sensorial e físico-química de Petit Gateau com recheio doce de banana com e sem casca.

\section{MATERIAL E MÉTODOS}

\section{Matéria-prima}

Foram desenvolvidas duas formulações de Petit Gateau sendo uma delas com o recheio do doce de banana com casca e a outra apenas o recheio do doce de banana.

Para as formulações propostas, utilizaram-se os seguintes ingredientes: banana com e sem casca; suco de laranja; farinha de trigo; achocolatado; margarina; fermento; açúcar; canela e ovo.

\section{Métodos}

\section{Formulações}

As formulações utilizadas para a elaboração do doce de banana com e sem casca estão descritas conforme Tabela 1. Para a elaboração da massa de Petit Gateau utilizando doce de banana com casca e o Petit Gateau utilizando apenas o doce de banana, às formulações seguem conforme Tabela 2, respectivamente.

Tabela 1. Formulação utilizada na elaboração do doce de banana com e sem casca.

\begin{tabular}{lcc}
\hline \multicolumn{1}{c}{ Ingredientes } & \% & Quantidade \\
\hline Bananas com ou sem casca & 57,47 & $500 \mathrm{~g}$ \\
Suco de laranja & 22,99 & $200 \mathrm{~mL}$ \\
Canela em pó & 1,15 & $10 \mathrm{~g}$ \\
Açúcar & 18,39 & $160 \mathrm{~g}$ \\
\hline
\end{tabular}

Tabela 2. Formulação utilizada na elaboração da massa de Petit Gateau com recheio de doce de banana com e sem casca.

\begin{tabular}{lcc}
\hline \multicolumn{1}{c}{ Ingredientes } & $\%$ & Quantidade \\
\hline Doce de banana com ou sem casca & 23,46 & $160 \mathrm{~g}$ \\
Achocolatado em pó & 1,76 & $12 \mathrm{~g}$ \\
Farinha de trigo & 17,60 & $120 \mathrm{~g}$ \\
Margarina & 14,66 & $100 \mathrm{~g}$ \\
Fermento & 1,47 & $10 \mathrm{~g}$ \\
Açúcar & 11,73 & $80 \mathrm{~g}$ \\
Ovos & 29,32 & $200 \mathrm{~g}$ \\
\hline
\end{tabular}

\section{Processamento do Petit Gateau}

Os recheios e as massas de Petit Gateau foram elaborados na Universidade Federal de Campina Grande (UFCG), Campus Pombal, conforme descrição do fluxograma apresentado na Figura 1 e na Figura 2.

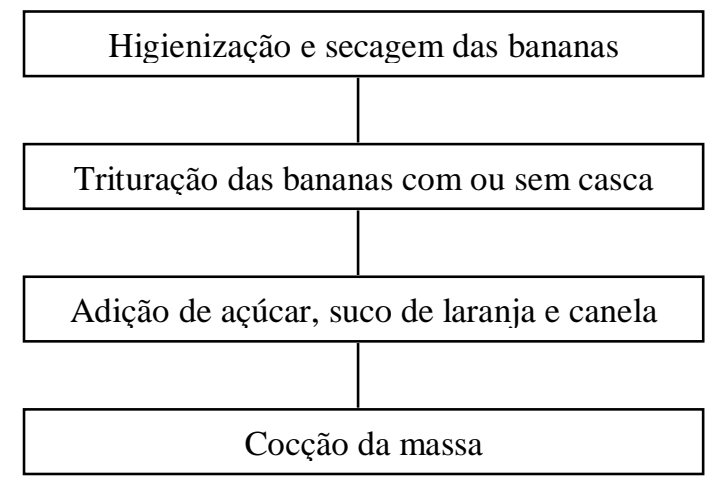

Figura 1. Fluxograma para a elaboração do doce banana com casca ou sem casca. CCTA//UFCG, Pombal-PB, 2015. 


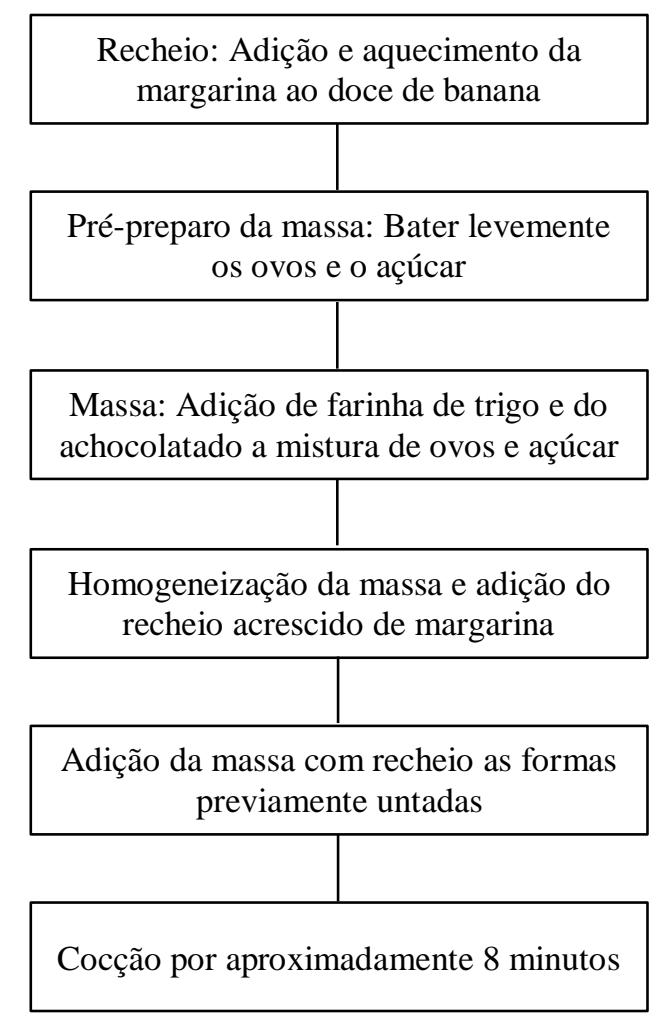

Figura 2. Fluxograma para a elaboração do Petit Gateau com recheio de doce de banana com ou sem casca. CCTA//UFCG, Pombal-PB, 2015.

\section{Análises Microbiológicas}

As análises microbiológicas foram realizadas no laboratório de Microbiologia de Alimentos da Universidade Federal de Campina Grande (UFCG), Campus Pombal, de acordo com as diretrizes gerais da Resolução RDC $\mathrm{n}^{\circ} 12$, de 02 de janeiro de 2001 da ANVISA, anexo I, grupo das farinhas, massas alimentícias, produtos para e de panificação, (industrializados e embalados) e similares. Sendo estabelecidos, valores toleráveis para Coliformes totais a $45^{\circ} \mathrm{C}$ e ausência de Salmonella $\mathrm{sp} / 25 \mathrm{~g}$. Os métodos de análise utilizados foram conforme descrito por SILVA et al. (2010).

\section{Análise Sensorial}

As duas formulações de Petit Gateau foram processadas $24 \mathrm{~h}$ antes da avaliação sensorial e cada assadeira individualmente, foi embaladas com filme de PVC e refrigeradas com temperatura média de $8^{\circ} \mathrm{C}$ até momentos antes da análise. Para a análise sensorial, os Petit Gateau foram submetidos ao aquecido, por 30 segundos, em forno micro-ondas e servidos quentes.

A aceitabilidade das duas formulações foi avaliada no laboratório de Analise Sensorial da Universidade Federal de Campina Grande (UFCG), Campus Pombal, contando com a participação de 60 provadores não treinados, recrutados dentre alunos e funcionários da instituição.

Os testes sensoriais foi realizado em cabines individuais e sob luz branca. A cada provador, foi entregue duas fichas, a primeira para o recrutamento e a segunda, para a avaliação sensorial do Petit Gateau. A equipe de aplicação do teste sensorial responsabilizou-se pela orientação geral dos provadores assim como, pelo atendimento individual a cada provador quando surgiram indagações do preenchimento e avaliação sensorial.

Durante o procedimento de análise sensorial foi avaliado a aceitação global e a aceitação dos atributos de aparência, sabor, textura e flavor, empregando-se uma escala hedônica de nove pontos. Na mesma ficha, foi incluso uma escala para avaliar a atitude do consumidor numa situação hipotética de compra do produto. Sendo assim, a cada provador foi solicitado à indicação da amostra que "mais gostou" e a que "menos gostou".

\section{Análises físico-químicas}

As análises físico-químicas foram realizadas no laboratório de Analise de Alimentos da Universidade Federal de Campina Grande (UFCG), Campus Pombal, conforme as metodologias descritas abaixo.

Umidade

$\mathrm{O}$ teor de umidade foi determinado em estufa a $105^{\circ} \mathrm{C}$ até peso constante, segundo as normas Analíticas do Instituto Adolfo Lutz (2008).

\section{Cinzas}

O teor de cinzas foi determinado pela incineração em mufla a $550^{\circ} \mathrm{C}$, segundo as normas Analíticas do Instituto Adolfo Lutz (2008)

\section{Lipídeos}

Os teores de lipídeos foram determinados através da extração contínua pelo Método Soxlhet, segundo as normas Analíticas do Instituto Adolfo Lutz (2008).

\section{Acidez Titulável}

A acidez titulável foi determinada segundo as normas Analíticas do Instituto Adolfo Lutz (2008).

\section{Sólidos solúveis (SS)}

Os teores de sólidos solúveis foram estimados a partir de 1 grama da amostra macerada em $2 \mathrm{ml}$ de água destilada. Após a maceração, a amostra foi filtrada em algodão e analisada em refratômetro digital com compensação automática de temperatura, foi determinada segundo as normas Analíticas do Instituto Adolfo Lutz (2008).

$\mathrm{pH}$

O potencial hidrogeniônico foi estimado a partir de 1 grama da amostra diluída em $10 \mathrm{ml}$ de água destilada. Após a diluição, a amostra foi homogeneizada e analisada através da leitura direta em potenciômetro digital, segundo as normas Analíticas do Instituto Adolfo Lutz (2008).

\section{Textura}

A análise de textura foi realizada com o auxílio de texturômetro digital.

\section{RESULTADOS E DISCUSSÃO}

\section{Análises Microbiológicas}

Na caracterização microbiológica das amostras de Petit Gateau, observou-se que tanto o produto sem casca (Tabela 
3), como o com casca, apresentaram valores desejáveis $\mathrm{n}^{\circ} 12$, de 2 de Janeiro de 2001, da Agência Nacional de Vigilância Sanitária (ANVISA).

Tabela 3. Resultados da analise microbiológica de Petit Gateau com recheio de doce de banana com e sem casca.

\begin{tabular}{|c|c|c|c|}
\hline Parâmetros & Padrão & $\begin{array}{c}\text { Amostra } \\
\text { s/ casca }\end{array}$ & $\begin{array}{c}\text { Amostra c/ } \\
\text { casca }\end{array}$ \\
\hline Coliformes a $35^{\circ} \mathrm{C}$ & - & $0 \mathrm{NMP} / \mathrm{g}$ & $0,3 \mathrm{NMP} / \mathrm{g}$ \\
\hline Coliformes a $45^{\circ} \mathrm{C}$ & $10^{2}$ & Ausente & Ausente \\
\hline Salmonella $\mathrm{sp} / 25 \mathrm{~g}$ & Ausente & Ausente & Ausente \\
\hline
\end{tabular}

Os coliformes a $35^{\circ} \mathrm{C}$, em termos da lei, os mesmos não estão dentro dos padrões, observa-se que a amostra sem casca não obteve contaminação pelos tais, enquanto que a com casca apresentou tal requisito em quantidade pequena, passível de aceitação. Para a última, tal circunstância é compreensível, haja vista que alguns fatores como a ambiência em que as frutas foram adquiridas e o contato direto tanto por parte do vendedor como de outros compradores, para com as frutas com casca, podem ter vindo a contribuir para com a contaminação das mesmas. Vale ressaltar que o processo de sanitização aplicado tanto a matéria-prima como aos utensílios fez-se muito válido, levando-se em consideração a possibilidade do mesmo ter diminuído a contaminação pelos microrganismos da categoria citada.

Em termos da patogenicidade, as amostras tanto sem casca como com casca mostraram estar em conformidade com a legislação vigente, apresentando ausência tanto de coliformes a $45^{\circ} \mathrm{C}$ como de Salmonella sp. Tal condição reforça a higiene com a qual o processo de preparação das mesmas foi conduzido, por parte dos manipuladores, novamente sendo válido citar, o processo de sanitização prévia empregado, confirmando assim o efeito benéfico das etapas de processo sob o resultado final.

\section{Análise Sensorial}

Na Tabela 4 estão apresentados os resultados estatísticos obtidos em relação a todos os atributos avaliados nas duas formulações de Petit Gateau. De acordo com os dados obtidos, observa-se que não houve diferença significativa entre as amostras de Petit Gateau com recheio de doce de banana, sem e com casca. Isso demonstra que o uso do doce de banana com casca pode ser um potencial alternativo para o melhor aproveitamento da fruta. Além de agregar maior valor econômico ao produto.

Tabela 4. Média dos resultados estatísticos obtidos em relação a todos os atributos avaliados nas duas formulações de Petit Gateau.

\begin{tabular}{llllll}
\hline & Aparência & Flavor & Sabor & Textura & $\begin{array}{c}\text { Op. } \\
\text { Global }\end{array}$ \\
\hline SC* $^{*}$ & $7.67 \mathrm{a}$ & $7.53 \mathrm{a}$ & $7.67 \mathrm{a}$ & $7.58 \mathrm{a}$ & $7.73 \mathrm{a}$ \\
CC $^{* *}$ & $7.33 \mathrm{a}$ & $7.43 \mathrm{a}$ & $7.67 \mathrm{a}$ & $7.13 \mathrm{a}$ & $7.33 \mathrm{a}$ \\
\hline
\end{tabular}

As médias seguidas pela mesma letra, na coluna, não diferem estatisticamente entre si, pelo Teste de Tukey ao nível de 5\% de probabilidade;

* Petit Gateau com recheio de doce de banana sem casca;

** Petit Gateau com recheio de doce de banana com casca; levando-se em consideração, o que é preconizado pela RDC

A preferência dos provadores, $56,7 \%$ apontaram o Petit Gateau com recheio de doce de banana sem casca como sendo a amostra que mais gostaram. Os demais provadores, 43,3\%, afirmaram ter gostado do Petit Gateau com recheio de doce de banana com casca. Mostrando assim, que o uso de doce de banana com casca pode ser utilizado sem grande comprometimento da aceitação do produto pelo consumidor, refletindo provavelmente apenas uma condição de costume ou de exposição frequente, deste, no mercado.

A Figura 3 apresenta na forma de histograma, a aceitação, indiferença e rejeição dos provadores em relação a cada parâmetro avaliado. Para todas as características avaliadas houve um nível de aceitação acima de $70 \%$, tanto para os Petits Gateaus preparados com recheio de doce de banana, com casca quanto sem casca.
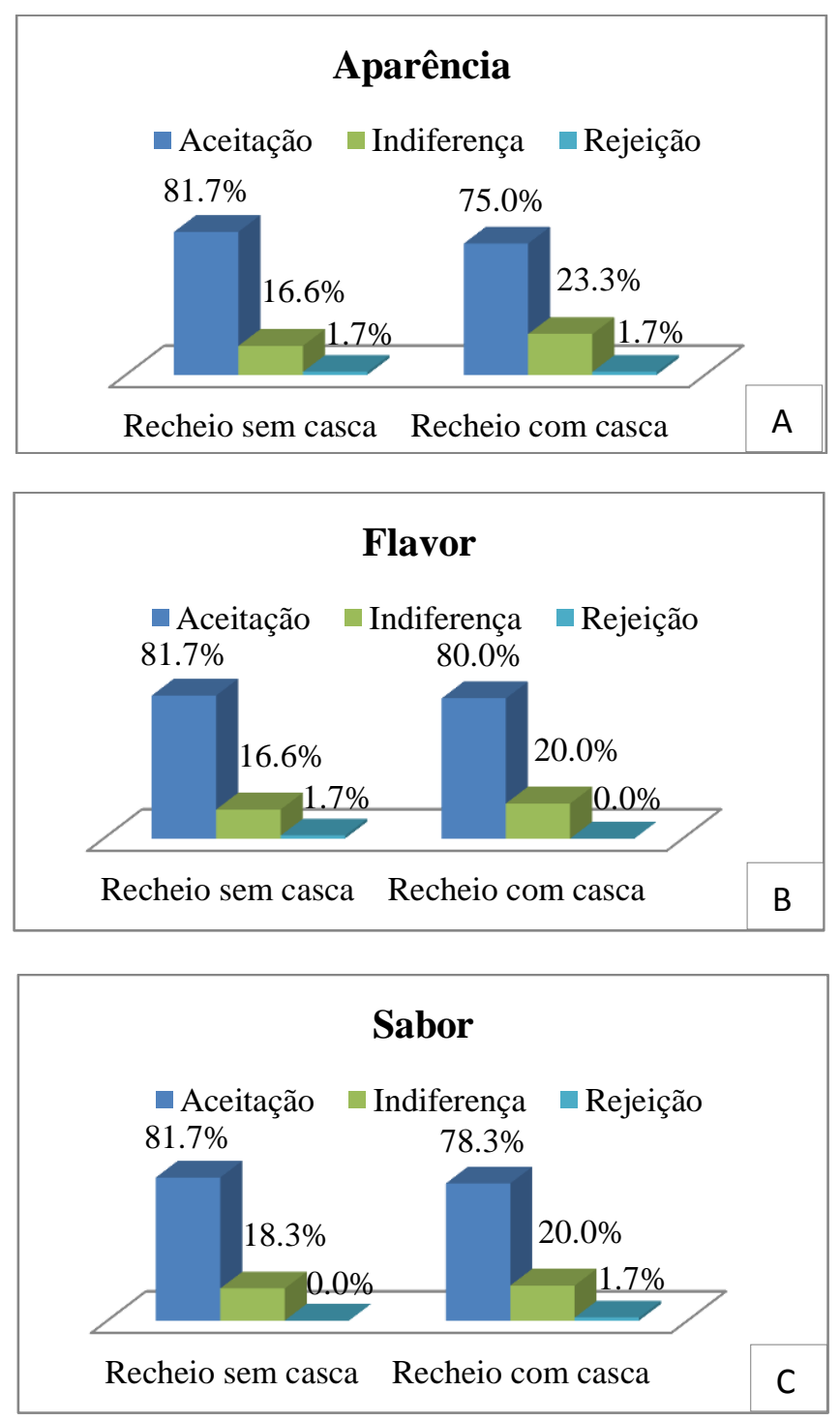

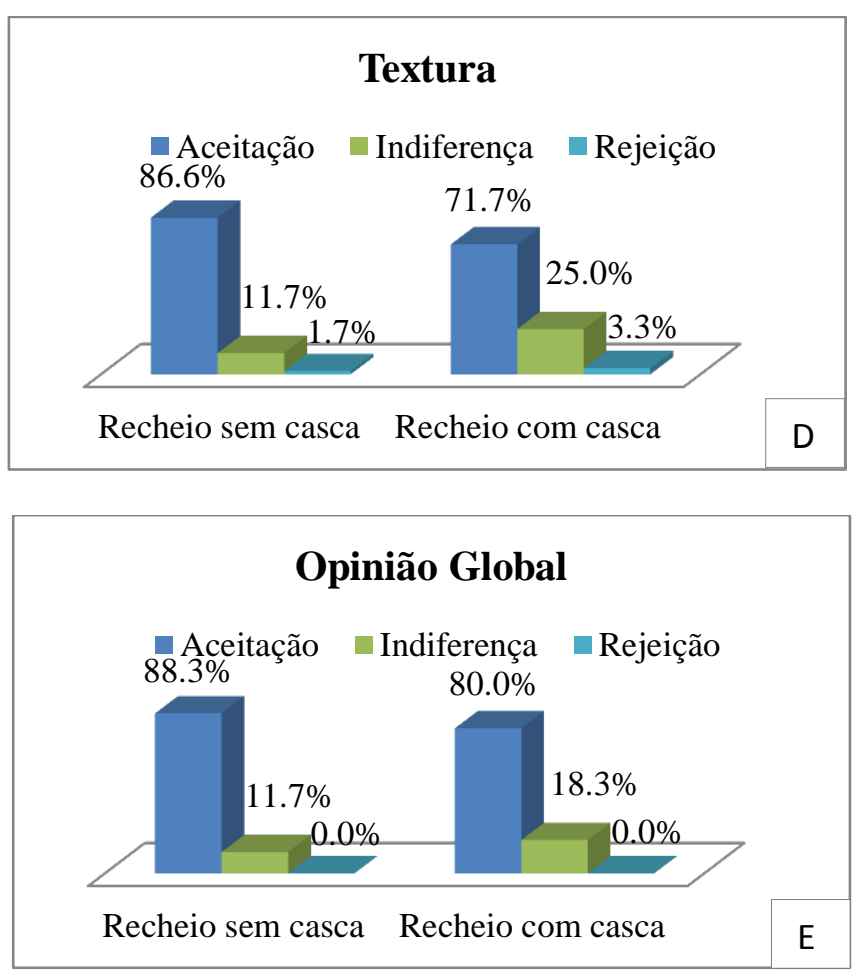

Figura 3. Histogramas de aceitação, indiferença e rejeição das características aparência (A), flavor (B), sabor (C), textura (D) e opinião global (E) de Petit Gateau, com recheio de doce de banana, sem e com casca. CCTA//UFCG, PombalPB, 2015.

A intenção de compra dos provadores de Petit Gateau ficou estimada em cerca de $60 \%$ (Figura 4), embora este tipo de produto não seja de consumo comum no dia-a-dia das pessoas.

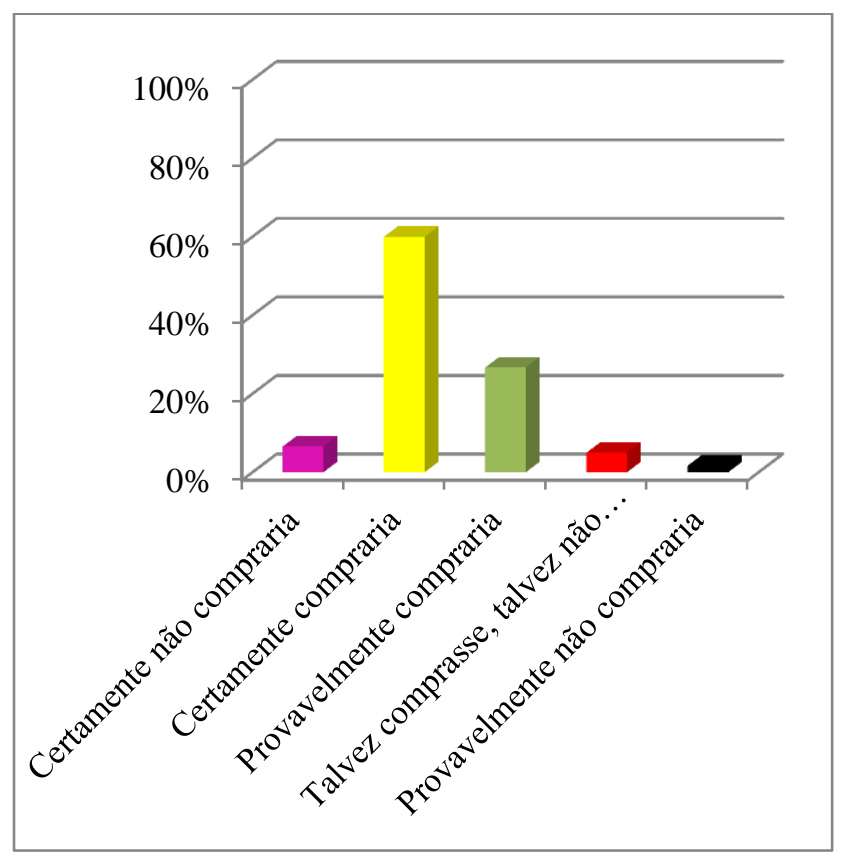

Figura 4. Média da intenção de compra do Petit Gateau por parte dos provadores. CCTA//UFCG, Pombal-PB, 2015.

\section{Análises físico-químicas}

Nos resultados obtidos, tem se que as formulações com casca e sem casca não diferiram significativamente entre si para os parâmetros $\mathrm{pH}$, lipídeos e acidez. Porém, o conteúdo de umidade, cinzas, ${ }^{\circ}$ Brix e textura, houve diferença significativa entre as amostras, pois os valores não apresentaram-se tão próximos.

Em função da escassez na literatura de fontes para uma comparação mais efetiva com o produto desenvolvido em questão (Petit Gateau), utilizou-se de trabalhos vinculados ao mesmo segmento do tal, ou seja, panificação.

Fasolin et al., (2007) ao estudarem a elaboração de três formulações distintas de biscoitos do tipo cookie com farinha da casca da banana, obtiveram valores para o tipo 1, para cinzas e lipídeos, respectivamente de 1,59\% e 19,11\%, fazendo-se, em relação ao conteúdo mineral inferior às amostras de Petit Gateau, e superior no teor lipídico também em relação as mesmas.

Estudo semelhante foi realizado por Santos (2013) ao elaborar uma formulação de biscoito tipo cookie a partir da substituição percentual da farinha de trigo por farinha de casca de abóbora, obtendo valores para cinzas e lipídeos respectivamente de $1,14 \%$ e $12,64 \%$. Observa-se que também neste caso os conteúdos minerais e de gordura também apresentaram-se inferiores aos das amostras de Petit Gateau. Tal condição decorrer possivelmente da diferença nas formulações empregadas, principalmente em ingredientes, por se tratarem de produtos diferenciados e pela fonte de resíduos utilizada, também diferentes.

Uchôa (2007) ao promover a adição de pós-alimentícios obtidos de resíduos de frutas tropicais na formulação de biscoitos, obteve, para o pó a partir do resíduo de caju, valores para cinzas e lipídeos respectivamente de 1,1\% e $15,4 \%$. Para o pó a partir do resíduo da goiaba, os valores encontrados foram de 1,2\% para o resíduo mineral e $16,1 \%$ para o conteúdo de gordura.

Obteve-se valor umidade (Tabela 5) superior apresentado pela amostra sem casca em relação à amostra com casca em função do recheio para a primeira ser preparado a partir da polpa da banana, que é mais úmida em relação ao resíduo.

Valores obtidos de ${ }^{\circ}$ Brix corroboram aos valores obtidos nos parâmetros anteriores considerando conteúdo nutricional da casca, a quantidade de sólidos solúveis, especialmente no que diz respeito aos açúcares, especialmente a frutose, o conteúdo para os mesmos na polpa se faz bem maior em relação à casca.

$\mathrm{O}$ pH para as duas formulações apresentou-se dentro da faixa de neutralidade, o que garante uma melhor estabilidade para o produto, principalmente em características tal como o sabor.

Os aspectos de textura e acidez, embora tenham apresentado valores relativamente baixos, porém desejáveis, reforçam a boa qualidade do produto elaborado, com a real possibilidade de inserção do mesmo junto ao mercado consumidor.

Tais parâmetros, possivelmente, podem ter passado alguma interferência da composição para o Petit Gateau e o recheio, além das condições de formulação e processo (recheio com e sem casca). 
Tabela 5. Resultados das análises físico-químicas de Petit Gateau com recheio de doce de banana com casca (A) e Petit Gateau com recheio de doce de banana sem casca (B).

\begin{tabular}{cccccccc}
\hline & Umidade, $\%$ & Cinzas, \% & $\mathrm{pH}$ & ${ }^{\circ}$ Brix & Acidez, \% & Lipídios, \% & Textura, N \\
\hline A & $62,84 \pm 0,80$ & $6,98 \pm 0,03$ & $7,77 \pm 0,14$ & $9,4 \pm 0,7$ & $0,55 \pm 0,12$ & $17,94 \pm 2,95$ & $2,6 \pm 0,20$ \\
B & $65,55 \pm 2,97$ & $7,53 \pm 0,37$ & $7,66 \pm 0,25$ & $14,5 \pm 1,2$ & $0,55 \pm 0,10$ & $16,01 \pm 2,75$ & $1,5 \pm 0,03$ \\
\hline
\end{tabular}

\section{CONCLUSÕES}

O Petit Gateau preparado com recheio de doce de banana com casca pode ser aproveitado na indústria de alimentos como forma de minimizar desperdícios, reduzir custos e propiciar melhor qualidade nutricional do alimento. Logo, o Petit Gateau com recheio de casca de banana pelo estudo realizado deve ser considerado um alimento alternativo.

\section{REFERÊNCIAS BIBLIOGRÁFICAS}

ANVISA - CNNPA - Comissão Nacional de Normas e Padrões para Alimentos. Resolução no 38 de 1977. Diário Oficial da União; Poder Executivo, de 27 de dezembro de 1977.

EMBRAPA. A cultura da banana. Brasília, DF: Editora Embrapa-SPI, 1997, p. 9-10.

FARFAN, J.M. Alimentação alternativa: análise crítica de uma proposta de intervenção nutricional. Cadernos de Saúde Pública, Rio de Janeiro, 14 (1): 205-212, jan-mar, 1998.

FASOLIN, L.H.; ALMEIDA, G.C.; CASTANHO, P.S.; NETTO-OLIVEIRA, E.R. Biscoitos produzidos com farinha de banana: avaliações química, física e sensorial. Ciência e Tecnologia de alimentos, Campinas, 27 (3): 524-529, jul.-set. 2007.

GONDIM, J.A.M.; MOURA, M.F.; DANTAS, A.F.; MEDEIROS, R.L.S.; SANTOS, K.M. Composição Centesimal e de minerais em cascas de frutas. Ciência e Tecnologia de Alimentos, 25 (4): 825-827, out.-dez. 2005.

Jornal UNESP, Julho/2006 - Ano XX - no 213. Disponível em: http://www.unesp.br/aci/jornal/213/desperdicio.php. Acessado em 08 de novembro de 2014.

INSTITUTO ADOLFO LUTZ. Normas analíticas do Instituto Adolfo Lutz: métodos químicos e físicos para análise de alimentos. São Paulo, 2008.

SANTOS, D.A. M. Formulação de biscoito tipo cookie a partir da substituição percentual de farinha de trigo por farinha de casca de abóbora (curcubita maxima) e albedo de maracujá amarelo (passiflora edulis flavicarpa). Dissertação de Mestrado - Programa de Pós Graduação em Alimentos e Nutrição - da Universidade Federal do Estado do Rio de Janeiro, como requisito parcial para obtenção do título de mestre em Alimentos e Nutrição. Rio de Janeiro-RJ, 2013.

UCHÔA, A.M.A. Adição de pós-alimentícios obtidos de resíduos de frutas tropicais na formulação de biscoitos. Dissertação submetida à Coordenação do curso de PósGraduação em Tecnologia de Alimentos, da Universidade Federal do Ceará, como requisito parcial para obtenção do grau de Mestre em Tecnologia de Alimentos. FortalezaCE, 2007. 